\title{
Importância da avaliação nutricional de recém-nascidos pré-termo por meio de relações antropométricas
}

\author{
The importance of the nutritional assessment of premature newborn infants by anthropometric \\ relationships
}

Laura Emília B. Cardoso', Mário Cícero Falcão²

RESUMO

Objetivo: Mostrar a importância das relações antropométricas na avaliação nutricional de prematuros de muito baixo peso (RNPTMBP), durante o primeiro mês de vida.

Métodos: Estudo longitudinal prospectivo incluindo RNPTMBP (peso ao nascer $<1.500 \mathrm{~g}$ e idade gestacional $<37$ semanas). Foram aferidos peso $(\mathrm{P})$, comprimento $(\mathrm{C})$ e perímetros braquial (PB) e cefálico (PC) e calculadas as relações antropométricas: índice ponderal (IP), índice de massa corporal (IMC) e razão PB/PC. A seguir, calcularam-se médias e desvios padrão das relações antropométricas, bem como a correlação entre parâmetros e relações antropométricas por meio do coeficiente de correlação de Pearson, nos três períodos do estudo (ao nascimento, 14 e 28 dias).

Resultados: Foram selecionados 55 recém-nascidos $(54,5 \%$ do sexo feminino), com $\mathrm{P}$ ao nascer de 1.076,70 $\pm 286,70 \mathrm{~g}$ e idade gestacional de $30,70_{ \pm} 2,10$ semanas. O IP era, ao

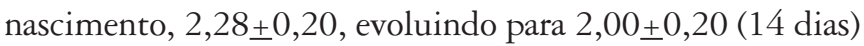
e $2,17 \pm 0,20$ aos 28 dias. O IMC mostrou: $8,20 \pm 1,10$ (nasci-

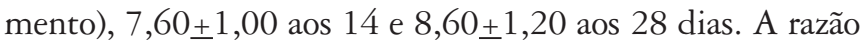
$\mathrm{PB} / \mathrm{PC}$ encontrada foi de $0,21 \pm 0,02$ ao nascimento, $0,19 \pm 0,02$ aos 14 e 0,21 $\pm 0,02$ aos 28 dias. Dentre as correlações, o IP foi o de menor correlação, seguida pela razão PB/PC. O IMC apresentou alta correlação com os parâmetros antropométricos.

Conclusões: IP, IMC e razão PB/PC apresentaram aumentos significantes entre 14 e 28 dias de vida, porém esta diferença não foi observada entre o nascimento e o $28^{\circ}$ dia. Dentre as relações antropométricas, o IMC mostrou-se mais fidedigno, pois demonstrou crescimento real dos prematuros após a segunda semana, por ter superado os valores do nascimento.

Palavras-chave: avaliação nutricional; antropometria; prematuro.

'Mestre em Ciências da Saúde pelo Departamento de Pediatria da Faculdade de Medicina da Universidade de São Paulo (FMUSP)

${ }^{2}$ Doutor em Pediatria pela FMUSP e professor colaborador do Departamento de Pediatria. Disciplina de Neonatologia da FMUSP

Endereço para correspondência:

Mário Cícero Falcão

Rua Vieira de Moraes, 45, apto. 51

\section{ABSTRACT}

Objective: To show the importance of the anthropometrical relationships in the nutritional assessment of premature infants, during the first month of life.

Methods: Longitudinal prospective study of premature infants (birth weight $<1,500 \mathrm{~g}$ and gestational age $<37$ weeks). Weight, length, cephalic circumference (CC), and brachial circumference (BC) were measured. The following anthropometrical relationships were calculated (average and standard deviation): ponderal index (PI), body mass index (BMI) and the BC/CC ratio. The correlation between anthropometrical parameters and the anthropometrical relationships were studied by Pearson coefficient in the three study periods (birth, 14 and 28 days).

Results: Fifty-five premature newborn infants were included ( $54.5 \%$ female): birth weight $1,076.70 \pm 286.70 \mathrm{~g}$ and gestational age $30.0 \pm 2.10$ weeks. The PI was, at birth, $2.28 \pm 0.20$, evolving to $2.00 \pm 0.20$ ( 14 days) and $2.17 \pm 0.20$ on the $28^{\text {th }}$ day. The BMI was $8.20 \pm 1.10$ (birth), $7.60 \pm 1.00$ on the $14^{\text {th }}$ and $8.60 \pm 1.20$ on the $28^{\text {th }}$ day. The $\mathrm{BC} / \mathrm{CC}$ ratio was $0.21 \pm 0.02$ at birth, $0.19 \pm 0.02$ on the $14^{\text {th }}$ and $0.21 \pm 0.02$ on the $28^{\text {th }}$ day. Amongst the correlations, the PI showed the least correlation, followed by the $\mathrm{BC} / \mathrm{CC}$ ratio. The BMI presented high correlation with the anthropometrical parameters.

Conclusions: PI, BMI and the BC/CC ratio presented significant increases between the $14^{\text {th }}$ and the $28^{\text {th }}$ day of life, but not between birth and $28^{\text {th }}$ day. The BMI was the most reliable anthropometrical relationship, since it showed a real growth after the second week, surpassing birth values.

Key-words: nutrition assessment; anthropometry; infant, premature.
CEP 04617-010 - São Paulo/SP
E-mail: profmariofalcao@yahoo.com.br

Projeto de Pesquisa financiado pela Fundação de Amparo à Pesquisa do Estado de São Paulo (Fapesp), processo 01/02700-2, aprovado em 21 de março de 2001

Recebido em: 21/11/2006

Aprovado em: 26/2/2007 


\section{Introdução}

A avaliação nutricional de recém-nascidos pré-termo de muito baixo peso (RNPTMBP) é tarefa importante em unidades de terapia intensiva neonatal, pois, neste período da vida, distúrbios do crescimento podem acarretar seqüelas em longo prazo. É possível realizar tal avaliação por meio da análise de parâmetros antropométricos, da comparação do crescimento com curvas de crescimento intra-uterino ou pós-natal e, também, pela análise de parâmetros bioquímicos ${ }^{(1)}$.

Dentre os parâmetros antropométricos, destacam-se: peso $(\mathrm{P})$, comprimento $(\mathrm{C})$, perímetros cefálico $(\mathrm{PC})$ e braquial (PB) e dobras cutâneas ${ }^{(1)}$. Além das medidas antropométricas, algumas relações entre parâmetros antropométricos foram descritas como formas de avaliação nutricional de recém-nascidos, objetivando ressaltar a proporcionalidade do crescimento intra-uterino e pós-natal, ou seja, tais relações se constituiriam em instrumentos para auxiliar na detecção da restrição do crescimento intra-uterino ${ }^{(2,3)}$.

$\mathrm{O}$ índice ponderal (IP) reflete a proporcionalidade do crescimento intra-uterino, pois leva em conta o peso e o cubo do comprimento. Sabe-se que um IP em torno de 2,00 correlaciona-se significativamente com a restrição do crescimento intra-uterino, o qual coloca o recém-nascido em risco de desenvolver, por exemplo, hipoglicemia no período neonatal imediato $^{(4)}$. Ademais, o IP revela-se bastante útil para diferenciar os recém-nascidos pequenos para a idade gestacional simétricos ou proporcionados dos assimétricos ou desproporcionados. Tal diferenciação apresenta grande implicação clínica, porque os recém-nascidos simétricos são aqueles que sofreram agressão nutricional desde o início da gestação, com risco mais elevado de comprometimento neurológico e conseqüente seqüela futura.

A relação entre PB e PC avalia a proporcionalidade corpórea baseada no princípio da preservação relativa do crescimento cefálico comparado à perda muscular e de gordura, em períodos de nutrição marginal ${ }^{(5)}$. Em 1984, Georgieff et al mostraram que a relação entre a circunferência média do braço e a circunferência cefálica é forma confiável para distinguir recém-nascidos com e sem restrição do crescimento intra-uterino, independentemente de sua classificação em adequado ou pequeno para a idade gestacional ${ }^{(6)}$. De acordo com a curva padrão da relação entre $\mathrm{PB}$ e $\mathrm{PC}$, os valores da razão crescem com a idade gestacional, e recém-nascidos com desnutrição intra-uterina apresentariam valores dessa relação abaixo do percentil $5^{(7)}$.

O índice de massa corporal (IMC) é utilizado na avaliação nutricional de adolescentes e adultos, servindo inclusive para definir sobrepeso e obesidade ${ }^{(8)}$. Recentemente, este índice foi validado para crianças entre zero e 36 meses de idade ${ }^{(9-11)}$, sendo uma relação bastante útil na detecção da desproporcionalidade do crescimento, já que também tem sido utilizado como marcador de adiposida$\mathrm{de}^{(12)}$ ou como parâmetro de crescimento harmônico ${ }^{(13,14)}$. Nos últimos anos, o IMC tem sido estudado também em recém-nascidos, porém com poucos relatos em prematu$\operatorname{ros}^{(11,14)}$, necessitando de novas pesquisas para obtenção de uma curva padrão.

Pela importância do papel do neonatologista em detectar precocemente os desvios do crescimento normal e, assim, evitar as possíveis seqüelas, seria necessário dispor de métodos rápidos, simples e de baixo custo, que permitissem a avaliação seriada do estado nutricional do recém-nascido pré-termo. Os parâmetros antropométricos e/ou as relações antropométricas poderiam preencher tais critérios e, dentre esses parâmetros, seria ideal contar com um único para se proceder tal avaliação. Neste contexto, o presente estudo tem como objetivo mostrar que o emprego rotineiro das relações antropométricas (razão entre PB e PC, IP e IMC) na avaliação nutricional de recém-nascidos pré-termos, durante o primeiro mês de vida, é um instrumento importante para interpretar o seu crescimento.

\section{Métodos}

O presente estudo foi conduzido, de forma prospectiva, em uma coorte de RNPTMBP (peso de nascimento inferior a $1.500 \mathrm{~g}$ e idade gestacional inferior a 37 semanas), admitidos no Berçário Anexo à Maternidade da Divisão de Pediatria Neonatal do Instituto da Criança do Hospital das Clínicas da Faculdade de Medicina da Universidade de São Paulo (FMUSP), após consentimento dos pais, no período de 4/5/2001 a 2/10/2002.

O estudo foi aprovado pela Comissão de Pesquisa e Ética do Instituto da Criança do Hospital das Clínicas da FMUSP e pela Comissão de Ética para Análise de Projetos de Pesquisa (CAPPesq) da Diretoria Clínica do Hospital das Clínicas e da FMUSP.

Foram excluídos do estudo RNPTMBP que apresentavam condições que alterassem o crescimento fetal ou situações que pudessem interferir nas medidas antropométricas, a saber: malformações maiores, cromossomopatias, hidrocefalia, hidropisia 
fetal, infecções congênitas, filhos de mães diabéticas, uso de drogas de abuso pela mãe e uso materno de corticóide.

Além disso, só foram considerados para o estudo os recém-nascidos que no $14^{\circ}$ dia de vida estivessem recebendo, no mínimo, $100 \mathrm{kcal} / \mathrm{kg} / \mathrm{dia}$ e $2 \mathrm{~g} / \mathrm{kg} /$ dia de oferta protéica, incluindo nutrição parenteral e enteral, não importando o tipo de dieta enteral (leite materno ou fórmula). Em relação ao $28^{\circ}$ dia de vida, só permaneceram no estudo os recémnascidos que estivessem recebendo nutrição enteral plena, também sem levar em consideração o tipo de dieta enteral.

Ao término da reanimação, ainda no Centro Obstétrico, foi obtido o peso, em gramas, em balança Filizola ${ }^{\circledR}$ eletrônica (com aproximação de $5 \mathrm{~g}$ ), após tarar, descontando-se qualquer equipamento porventura atado ao recém-nascido durante as manobras de reanimação.

Nas primeiras 24 horas de vida, foram realizadas, pela própria pesquisadora, as medidas do $\mathrm{C}, \mathrm{PC}$ e $\mathrm{PB}$, no intervalo entre duas ofertas de dieta, caso o recém-nascido já estivesse recebendo dieta enteral.

O comprimento, em centímetros, foi obtido com o recém-nascido em decúbito dorsal, sobre superfície rígida por régua de madeira (com aproximação de $0,1 \mathrm{~cm}$ ), estando uma extremidade fixa (cefálica) e a outra móvel (podálica), com o auxílio de outra pessoa na contenção do recém-nascido, sendo considerada a média de três medidas consecutivas.

O PC, em centímetros, foi obtido por fita métrica inextensível (com aproximação de $0,1 \mathrm{~cm}$ ), levando-se em conta o maior diâmetro occipitofrontal, sendo considerada a média de três medidas consecutivas.

$\mathrm{O} \mathrm{PB}$, em centímetros, foi obtido no ponto médio entre o acrômio e o olécrano do braço esquerdo estendido e mão em posição prona, com fita métrica inextensível (com aproximação de $0,1 \mathrm{~cm}$ ), sendo considerada a média de três medidas consecutivas.

A razão entre $\mathrm{PB}$ e $\mathrm{PC}(\mathrm{PB} / \mathrm{PC})$ foi determinada pelo cálculo matemático simples entre os dois valores obtidos no mesmo momento.

O cálculo do IP foi determinado pela fórmula: $\mathrm{IP}=$ peso $(\mathrm{g})$ $\mathrm{x}$ 100/ comprimento $(\mathrm{cm})^{3}$. O cálculo do IMC foi determinado pela fórmula: $\mathrm{IMC}=$ peso $(\mathrm{kg}) /$ comprimento $(\mathrm{m})^{2}$.

Para o cálculo da idade gestacional, foram utilizadas, por ordem de prioridade:

- Informação materna sobre a data da última menstruação (Regra de Naegele, que considera o tempo de gestação normal de 280 dias), quando esta diferia em, no máximo, duas semanas da idade gestacional fornecida pela ultrasonografia fetal realizada até a $20^{a}$ semana da gestação;
- Ultra-sonografia realizada até 20 semanas de gestação( ${ }^{(15)}$, nos casos em que a idade gestacional materna não foi considerada confiável e a diferença entre as idades calculadas pelos métodos ultra-sonográficos e o New Ballard ${ }^{(16)}$ foi inferior a duas semanas;

- Idade gestacional pós-natal calculada pelo Método de New Ballard $^{(16)}$, quando este diferia em mais de duas semanas das idades gestacionais materna e ultra-sonográfica.

A classificação dos recém-nascidos foi baseada nas curvas de referência de peso para a idade gestacional obtidas por Alexander et al, em $1996^{(17)}$, sendo considerados adequados para a idade gestacional os recém-nascidos situados entre os percentis 10 e 90 da curva, grandes para a idade gestacional aqueles situados acima do percentil 90, e pequenos para a idade gestacional aqueles situados abaixo do percentil 10 .

Os parâmetros antropométricos ( $\mathrm{P}, \mathrm{C}, \mathrm{PC}$ e $\mathrm{PB}$ ) foram medidos ao nascimento, no $14^{\circ}$ e $28^{\circ}$ dias de via. Da mesma forma, as relações antropométricas (PB/PC, IP e IMC) também foram calculadas ao nascimento, com 14 e 28 dias de vida.

Para a análise estatística, foram utilizados os programas Sigma Stat e Excel para a elaboração do banco de dados. Calcularam-se as médias e desvios padrão para as variáveis antropométricas (P, C, PC e PB) e para as relações antropométricas (PB/PC, IP e IMC) nos três períodos do estudo (ao nascimento, com 14 e 28 dias de vida). Para a análise comparativa entre os grupos, foi usado o teste ANOVA. As correlações entre os parâmetros antropométricos ( $\mathrm{P}, \mathrm{C}, \mathrm{PC}$ e $\mathrm{PB})$ e as relações antropométricas (PB/PC, IP e IMC) foram calculadas por meio do coeficiente de correlação de Pearson. Em todas as análises, considerou-se significante $p<0,05$.

\section{Resultados}

Os dados obtidos foram coletados entre 4/5/2001 e 2/10/2002. Foram incluídos 55 RNPTMBP que preencheram todos os critérios para análise. A Figura 1 mostra a seleção da população do estudo e a Tabela 1 apresenta as características da população estudada.

A Tabela 2 descreve as características antropométricas e bioquímicas da população estudada nos três períodos (ao nascimento, com 14 e 28 dias de vida). As Tabelas 3, 4 e 5 apresentam as correlações entre os parâmetros antropométricos $(\mathrm{P}$, C, PC e PB) e as relações antropométricas (PB/PC, IP e IMC), pelo coeficiente de correlação de Pearson, nos três períodos estudados (ao nascimento, com 14 e 28 dias de vida). 


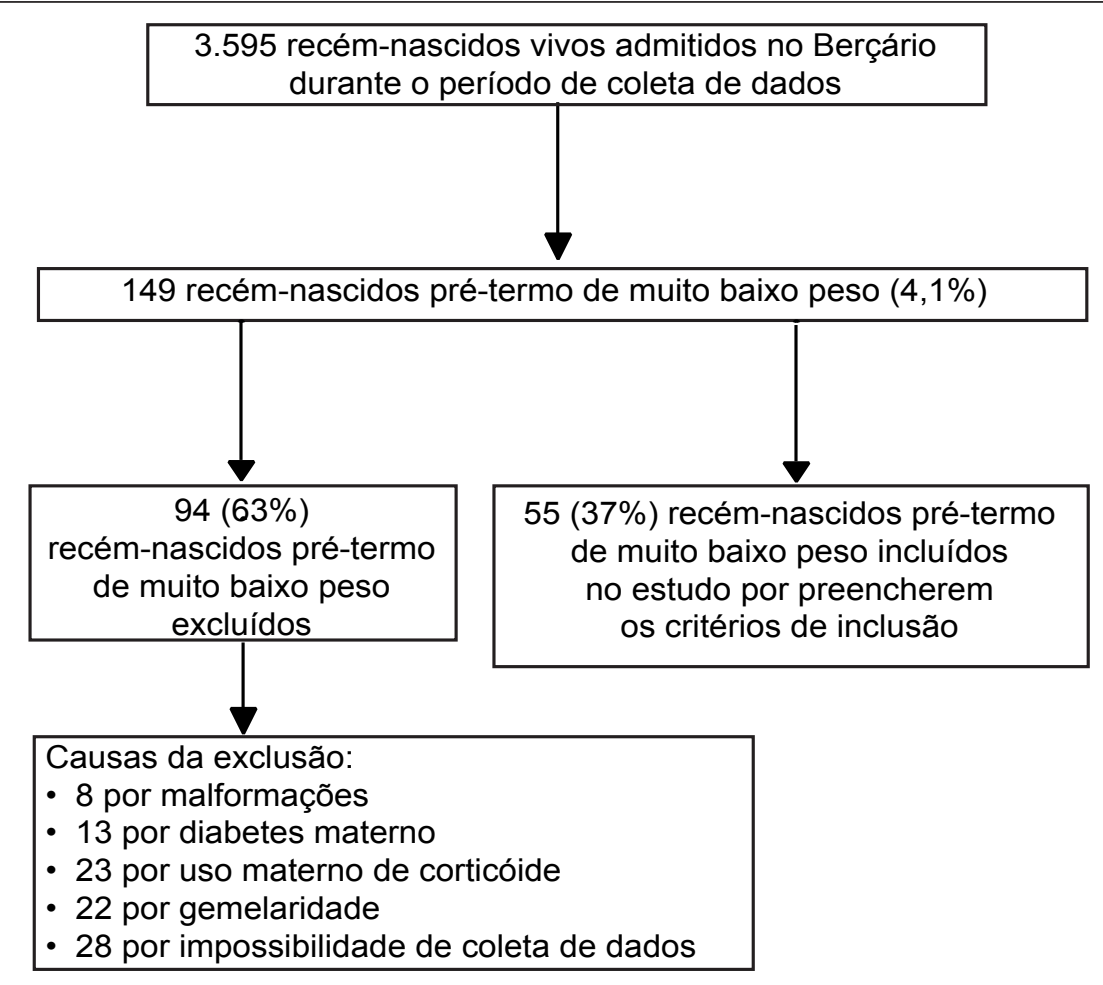

Figura 1 - Seleção da população do estudo

Tabela 1 - Características dos recém-nascidos pré-termo de muito baixo peso

\begin{tabular}{lc}
\hline Tamanho amostral & 55 \\
\hline Peso ao nascimento (g) & $1077 \pm 287$ \\
Idade gestacional (sem) & $30,7 \pm 2,1$ \\
Adequados para a idade gestacional & $17(31 \%)$ \\
Pequenos para a idade gestacional & $38(69 \%)$ \\
Sexo feminino & $30(54,5 \%)$ \\
\hline
\end{tabular}

Tabela 2 - Características antropométricas dos recém-nascidos pré-termos de muito baixo peso nos três períodos do estudo (ao nascimento, com 14 e 28 dias de vida)

\begin{tabular}{lccc}
\hline & Nascimento & 14 dias & 28 dias \\
\hline $\mathrm{P}(\mathrm{g})$ & $1.077 \pm 287$ & $1.095 \pm 310$ & $1.359 \pm 367^{*}$ \\
& $(500-1480)$ & $(600-1670)$ & $(750-2070)$ \\
$\mathrm{C}(\mathrm{cm})$ & $35,8 \pm 3,2$ & $37,6 \pm 3,2$ & $39,4 \pm 2,4^{*}$ \\
& $(28,0-41,0)$ & $(30,5-42,5)$ & $(32,0-44,5)$ \\
$\mathrm{PC}(\mathrm{cm})$ & $26,0 \pm 2,3$ & $26,9 \pm 2,4$ & $28,5 \pm 2,1^{*}$ \\
& $(21,0-30,0)$ & $(22,0-31,5)$ & $(24,5-33,0)$ \\
PB $(\mathrm{cm})$ & $5,5 \pm 0,7$ & $5,5 \pm 0,9$ & $6,1 \pm 0,9^{*}$ \\
& $(4,0-7,3)$ & $(3,8-7,0)$ & $(4,6-7,7)$ \\
PB/PC & $0,21 \pm 0,02$ & $0,19 \pm 0,02^{*}$ & $0,21 \pm 0,02$ \\
& $(0,16-0,25)$ & $(0,16-0,23)$ & $(0,17-0,24)$ \\
IP & $2,3 \pm 0,20$ & $2,0 \pm 0,2$ & $2,2 \pm 0,2^{*}$ \\
& $(1,6-3,0)$ & $(1,6-2,3)$ & $(1,7-2,6)$ \\
IMC & $8,2 \pm 1,1$ & $7,6 \pm 1,0 *$ & $8,6 \pm 1,2$ \\
& $(5,7-10,2)$ & $(5,8-9,6)$ & $(6,4-11,4)$ \\
\hline
\end{tabular}

*diferente estatisticamente em relação às demais colunas $(p<0,05)$ 
Tabela 3 - Correlação entre os parâmetros antropométricos e as relações antropométricas ao nascimento

\begin{tabular}{lcccccc}
\hline & \multicolumn{2}{c}{ PB/PC } & \multicolumn{2}{c}{ IP } & \multicolumn{2}{c}{ IMC } \\
\cline { 2 - 7 } & $\mathbf{r}$ & $\boldsymbol{p}$ & $\mathbf{r}$ & $\boldsymbol{p}$ & $\mathbf{r}$ & $\boldsymbol{p}$ \\
$\mathrm{P}$ & 0,38 & 0,004 & 0,31 & 0,02 & 0,85 & $<0,001$ \\
$\mathrm{C}$ & 0,35 & 0,009 & $-0,05$ & 0,68 & 0,61 & $<0,001$ \\
$\mathrm{PC}$ & 0,21 & 0,12 & 0,14 & 0,29 & 0,71 & $<0,001$ \\
$\mathrm{~PB}$ & 0,75 & $<0,001$ & 0,15 & 0,27 & 0,66 & $<0,001$ \\
\hline
\end{tabular}

$\mathrm{r}=$ coeficiente de correlação de Pearson entre as variáveis; $p=$ nível de significância; $\mathrm{PB} / \mathrm{PC}=$ razão entre os perímetros braquial e cefálico; $\mathrm{IP}=$ índice ponderal; IMC=índice de massa corporal

Tabela 4 - Correlação entre os parâmetros antropométricos e as relações antropométricas com 14 dias de vida

\begin{tabular}{lcccccc}
\hline & \multicolumn{2}{c}{ PB/PC } & \multicolumn{2}{c}{ IP } & \multicolumn{2}{c}{ IMC } \\
\cline { 2 - 7 } & $\mathbf{r}$ & $\boldsymbol{p}$ & $\mathbf{r}$ & $\boldsymbol{p}$ & $\mathbf{r}$ & $\boldsymbol{p}$ \\
$\mathrm{P}$ & 0,73 & $<0,001$ & 0,52 & $<0,001$ & 0,93 & $<0,001$ \\
$\mathrm{C}$ & 0,71 & $<0,001$ & 0,21 & $<0,001$ & 0,76 & $<0,001$ \\
$\mathrm{PC}$ & 0,56 & $<0,001$ & 0,51 & $<0,001$ & 0,87 & $<0,001$ \\
$\mathrm{~PB}$ & 0,88 & $<0,001$ & 0,49 & $<0,001$ & 0,88 & $<0,001$ \\
\hline
\end{tabular}

$r=$ coeficiente de correlação de Pearson entre as variáveis; $p=$ nível de significância; $\mathrm{PB} / \mathrm{PC}=$ razão entre os perímetros braquial e cefálico; $\mathrm{IP}=$ índice ponderal; IMC=índice de massa corporal

Tabela 5 - Correlação entre os parâmetros antropométricos e as relações antropométricas com 28 dias de vida

\begin{tabular}{lcccccc}
\hline & \multicolumn{2}{c}{ PB/PC } & \multicolumn{2}{c}{ IP } & \multicolumn{2}{c}{ IMC } \\
\cline { 2 - 7 } & $\mathbf{r}$ & $\boldsymbol{p}$ & $\mathbf{r}$ & $\boldsymbol{p}$ & $\mathbf{r}$ & $\boldsymbol{p}$ \\
\hline P & 0,78 & $<0,001$ & 0,59 & $<0,001$ & 0,93 & $<0,001$ \\
$\mathrm{C}$ & 0,73 & $<0,001$ & 0,27 & 0,08 & 0,74 & $<0,001$ \\
$\mathrm{PC}$ & 0,56 & $<0,001$ & 0,67 & $<0,001$ & 0,90 & $<0,001$ \\
$\mathrm{~PB}$ & 0,90 & $<0,001$ & 0,62 & $<0,001$ & 0,91 & $<0,001$ \\
\hline
\end{tabular}

$\mathrm{r}=$ coeficiente de correlação de Pearson entre as variáveis; $p=$ nível de significância; $\mathrm{PB} / \mathrm{PC}=$ razão entre os perímetros braquial e cefálico; $\mathrm{IP}=$ índice ponderal; $\mathrm{IMC}=$ índice de massa corporal

\section{Discussão}

A avaliação nutricional do recém-nascido é tarefa difícil e necessária em uma unidade de terapia intensiva neonatal, principalmente quando se trata de prematuros, uma vez que nasceram antes do período de maior crescimento somático e de depósitos de nutrientes, como glicogênio, proteínas, gorduras, vitaminas, microelementos e minerais. Diante desta assertiva, a avaliação nutricional torna-se peça fundamental na rotina diária dessas $\operatorname{crianças~}^{(1)}$.

A utilização de parâmetros antropométricos nessa avaliação é uma maneira relativamente simples, facilmente disponível e de baixo custo ${ }^{(18)}$. Entretanto, esses parâmetros necessitam de uma aferição precisa para terem validade, o que, por muitas vezes, é difícil em uma população de recémnascidos prematuros, especialmente nos de muito baixo peso. Quanto menor o recém-nascido, maior a necessidade de monitorá-lo com equipamentos atados ao corpo, interferindo na medida do $\mathrm{P}$, na precisão das medidas do $\mathrm{C}$ e dos $\mathrm{PC}$ e PB. Além disso, nos primeiros dias de vida ocorrem grandes modificações na composição corpórea, com redução significativa do espaço extracelular e conseqüente perda de peso, que pode alcançar $20 \%$ no recém-nascido pré-termo extremo ${ }^{(19)}$. Também o acúmulo de edema e a presença de malformações, com aumento ou redução das dimensões de partes do corpo, podem afetar de forma relevante o peso, independentemente dos aspectos nutricionais ${ }^{(1)}$. Dessa forma, o peso não deve ser utilizado de forma isolada na avaliação nutricional nas primeiras semanas de vida de recém-nascidos doentes ou imaturos.

$\mathrm{O}$ uso das relações antropométricas na avaliação nutricional dos RNPTMBP está descrito há vários anos ${ }^{(20)}$. É possível utilizar o IP e a razão entre PB e PC ${ }^{(21)}$, aplicando-se a curva de referência para esta relação descrita por Sasanow et al em $1986^{(5)}$. Nos últimos anos, alguns estudos passaram a incluir o IMC na avaliação de recémnascidos prematuros, porém ainda não existe um padrão de referência para esses neonatos ${ }^{(12,22)}$.

Wilcox, em $1983^{(3)}$, sugeriu que o uso de medidas que determinam a proporcionalidade corpórea, por exemplo, a relação entre $\mathrm{PB}$ e $\mathrm{PC}$ e a relação entre $\mathrm{P}$ e $\mathrm{C}$, podem ser mais úteis que medidas antropométricas isoladas para identificar recémnascidos de risco para complicações metabólicas precoces ${ }^{(5)}$.

A relação entre PB e PC é um bom indicador do estado protéico-calórico do recém-nascido e permite caracterizá-los quanto à restrição de crescimento intra-uterino; no entanto, a medida de $\mathrm{PB}$ não é realizada rotineiramente na prática clínica.

Já a medida de $\mathrm{P}$ ajustada pelo $\mathrm{C}$ representa um bom método de avaliação de composição corpórea, principalmente de adiposidade, e apresenta a vantagem de detectar alterações nutricionais mais facilmente, considerando o princípio fisiológico do organismo de poupar o crescimento do $\mathrm{C}$ em relação ao $\mathrm{P}$ nos casos de distúrbios nutricionais leves e moderados. Em conseqüência, vários índices derivados desta relação estão disponíveis ${ }^{(22)}$.

Dentre os índices freqüentemente utilizados na prática pediátrica, que correlacionam o peso e o comprimento elevado à potência " $\mathrm{n}$ ” $\left(\mathrm{P} / \mathrm{C}^{\mathrm{n}}\right)$, pode-se citar: $\mathrm{P} / \mathrm{C}$ e $\mathrm{P} / \mathrm{C}^{3}(\mathrm{IP})$. Entretanto, o " $n$ " elevado ao quadrado, ou seja, o IMC é o que melhor 
ajusta o $\mathrm{P}$ pelo $\mathrm{C}$, removendo a influência direta do aumento do $\mathrm{P}$ com a idade, observado quando "n" é igual a um, e, ao mesmo tempo, evitando elevar a medida de $\mathrm{C}$ à terceira potência, pois se sabe que a medida de C é mais difícil de ser obtida na prática diária e pode estar sujeita a erros, já que potências maiores elevam exponencialmente tais $\operatorname{erros}^{(23,24)}$.

Deste modo, o IMC, também conhecido como índice de Quetelet, constitui medida de proporcionalidade de extrema importância para avaliar o estado nutricional. Tal índice tem sido bastante utilizado em adultos como um indicador de obesidade, considerando sua boa correlação com a gordura corpórea total ${ }^{(8)}$, e já foi validado em crianças como bom marcador de adiposidade e sobrepeso, apresentando estreita correlação com outros parâmetros que avaliam a porcentagem de gordura corpórea, como as pregas cutâneas, a densitometria e a bioimpedância eletromagnética ${ }^{(11)}$. Além da validação do IMC como bom marcador de adiposidade em crianças, o interesse pelo seu uso cresceu à medida que se notou que o IMC mensurado na infância pode ser preditivo em relação ao IMC na idade adulta ${ }^{(13)}$. Guo et $a^{(8)}$ encontraram correlação positiva em adolescentes com altos valores de IMC e risco de sobrepeso e obesidade na idade adulta. Apesar de evidências da associação dos valores do IMC em crianças menores de dois anos e obesidade na adolescência e idade adulta, a sua relação com o período fetal e neonatal ainda não foi muito estudada ${ }^{(25)}$. Além disso, ainda não está totalmente estabelecido o comportamento ideal das relações antropométricas no período neonatal em RNPTMBP.

Pelo exposto, a comparação entre os três índices utilizados nesta pesquisa mostra-se importante para aplicação clínica, pois, além de serem obtidos facilmente, auxiliarão na interpretação do crescimento destas crianças. Em relação aos dados encontrados, é importante comentar que se observou redução significativa nos valores da razão entre PB e PC entre o nascimento $(0,21 \pm 0,02)$ e 14 dias de vida $(0,19 \pm 0,02)$, com recuperação aos 28 dias de vida $(0,21 \pm 0,02)$, conforme Tabela 2. A evolução do IP revela redução significativa de $2,28 \pm 0,2 \mathrm{~g} / \mathrm{cm}^{3}$ ao nascimento para $2,00 \pm 0,2 \mathrm{~g} / \mathrm{cm}^{3}$ aos 14 dias de vida e recuperação parcial para $2,17 \pm 0,2 \mathrm{~g} / \mathrm{cm}^{3}$ aos 28 dias de vida, ainda significativamente menor do que os valores do nascimento (Tabela 2). Em relação ao IMC, nota-se redução significativa de $8,2 \pm 1,1 \mathrm{~kg} / \mathrm{m}^{2}$ ao nascimento para
$7,6 \pm 1,0 \mathrm{~kg} / \mathrm{m}^{2}$ aos 14 dias de vida e posterior recuperação para $8,6 \pm 1,2 \mathrm{~kg} / \mathrm{m}^{2}$ aos 28 dias de vida (Tabela 2). Dessa forma, a análise das relações antropométricas revela que, aos 28 dias de vida, as proporções corpóreas são semelhantes às do nascimento, exceto a do IP. O uso do P nos cálculos do IP e do IMC determina declínio dos mesmos nas primeiras semanas, acompanhando o perfil do P. O mesmo efeito é esperado para a razão entre $\mathrm{PB}$ e $\mathrm{PC}$, com a possível preservação do crescimento encefálico.

Observaram-se também fracas correlações entre $\mathrm{P}(\mathrm{r}=0,38)$ e C $(r=0,35)$, com a razão entre PB e PC, embora significantes $(p<0,05)$, além da fraca correlação entre IP e P $(r=0,31)$. Entretanto, podem-se notar fortes correlações entre os parâmetros antropométricos (P, C, PC e PB) e o IMC, segundo Tabela 3. Aos 14 dias (Tabela 4), nota-se forte correlação de todos os parâmetros antropométricos (P, C, PC e PB) e a razão entre PB e PC. Neste período, o IP correlacionou-se positivamente com todos os parâmetros antropométricos, com exceção do C. Como no período anterior, o IMC correlacionou-se também de forma positiva com todos os parâmetros antropométricos estudados.

As correlações entre os parâmetros antropométricos e relações antropométricas aos 28 dias de vida são semelhantes às descritas para 14 dias de vida, com ausência de correlação apenas entre IP e C.

Estas análises sugerem que as relações antropométricas analisadas neste estudo, ou seja, a razão entre PB e PC, IP e IMC apresentam comportamento diferente nos três momentos avaliados. Dentre as três correlações, o IP foi o de menor correlação, seguida pela razão entre PB e PC. Já o IMC apresentou alta correlação com todos os parâmetros antropométricos avaliados, inclusive com PB e PC, que são independentes do seu cálculo, nos três períodos estudados. Além disso, o IMC mostrou-se mais fidedigno ao demonstrar o crescimento real dos RNPTMBP após a segunda semana de vida, superando os valores observados ao nascimento. Desta maneira, o IMC mostra-se promissor na avaliação nutricional de recém-nascidos ${ }^{(25)}$, inclusive prematuros, tanto ao nascimento como durante o primeiro mês de vida, podendo traduzir o crescimento harmônico dessas crianças, ou seja, tanto da massa gorda como da magra. 


\section{Referências bibliográficas}

1. Falcão MC, Cardoso LEMB. Avaliação nutricional do recém-nascido pré-termo. Rev Bras Nutr Clin 2001;16:144-7.

2. Falcão MC, Cardoso LEMB. Avaliação e monitorização nutricional. In: Feferbaum R, Falcão MC, editores. $1^{\text {a }}$ ed. Nutrição do recém-nascido. São Paulo: Atheneu; 2002. p.55-66.

3. Wilcox AJ. Intrauterine growth retardation: beyond birthweight criteria. Early Hum Dev 1983;8:189-93.

4. Lubchenco LO, Bard H. Incidence of hypoglycemia in newborn infants classified by birth weight and gestational age. Pediatrics 1971;47:831-8.

5. Sasanow SR, Georgieff MK, Pereira GR. Mid-arm circumference and mid-arm/ head circumference ratios: standard curves for anthropometric assessment of neonatal nutritional status. J Pediatr 1986;109:311-5.

6. Georgieff MK, Sasanow SR, Pereira GR. Mid-arm circumference/head circumference ratio $(\mathrm{MAC} / \mathrm{HC})$ for identification of intrauterine growth disorders in neonates. J Am Coll Nutr 1984;3:263-8.

7. Sasanow SR, Spitzer AR, Pereira GR, Heaf L, Watkins JB. Effect of gestational age upon prealbumin and retinol binding protein in preterm and term infants. J Pediatr Gastroenterol Nutr 1986;5:111-5.

8. Guo SS, Wu W, Chumlea WC, Roche AF. Predicting overweight and obesity in adulthood from body mass index values in childhood and adolescence. Am J Clin Nutr 2002;76:653-8.

9. Haschke F, van't Hof MA. Euro-growth references for body mass index and weight for length. J Pediatr Gastroenterol Nutr 2000;31:S48-S59.

10. Karlberg J, Luo ZC, Albertsson-Wikland K. Body mass index references values (mean and SD) for Swedish children. Acta Paediatr 2001; 90:1427-34.

11. Tanaka T, Matsuzaki A, Kuromaru R, Kinukawa N, Nose $Y$, Matsumoto $T$ et al. Association between birth weight and body mass index at 3 years of age. Pediatr Int 2001;43:641-6.

12. Dietz WH, Bellizzi MC. Introduction: the use of body mass index to assess obesity in children. Am J Clin Nutr 1999;70:123S-5S.

13. Lynch J, Wang XL, Wilcken DE. Body mass index in Australian children: recent changes and relevance of ethnicity. Arch Dis Child 2000;82:16-20.
14. Parsons TJ, Power C, Manor O. Fetal and early life growth and body mass index from birth to early adulthood in 1958 British cohort: longitudinal study. BMJ 2001;323:1331-5.

15. Hadlock FP, Deter RL, Harrist RB, Park SK. Estimating fetal age: computer assisted analysis of multiple fetal growth parameters. Radiology 1984;154:497-501.

16. Ballard JL, Khoury JC, Wedig K, Wang L, Eilers-Walsman BL, Lipp R. New Ballard Score, expanded to include extremely premature infants. J Pediatr 1991;119:417-23

17. Alexander GR, Himes JH, Kaufman RB, Mor J, Kogan M. A United States national reference for fetal growth. Obstet Gynecol 1996;87:163-8.

18. Anderson DM. Nutritional assessment and therapeutic interventions for the preterm infant. Clin Perinatol 2002;29:313-26.

19. Georgieff MK, Sasanow SR. Nutritional assessment of the neonate. Clin Perinatol 1986;13:73-90.

20. Ramos JLA. Avaliação do crescimento intra-uterino por medidas antropométricas do recém-nascido [tese de doutorado]. São Paulo (SP): USP; 1983.

21. Georgieff MK, Sasanow SR, Chockalingam UM, Pereira GR. A comparison of the mid-arm circumference/head circumference ratio and ponderal index for the evaluation of newborn infants after abnormal intrauterine growth. Acta Paediatr 1988;77:214-9.

22. Abrantes MM, Lamounier JA, Colosimo EA. Recommendations for the use of body mass index for the classification of overweight and obese children and adolescents. Food Nutr Bull 2002;23:262-6.

23. Gill A, Yu VY, Bajuk B, Astbury J. Postnatal growth in infants born before 30 weeks' gestation. Arch Dis Child 1986;61:549-53.

24. Must A, Dallal GE, Dietz WH. Reference data for obesity: $85^{\text {th }}$ and $95^{\text {th }}$ percentiles of body mass index (wt/ht2) and triceps skinfold thickness. Am J Clin Nutr 1991;53:839-46.

25. Brock RS, Falcão MC, Leone C. Body mass index references values for newborn according to gestational age. Clin Nutr 2004,23:A766. 